\title{
Biomass Morphology Subjected to Different Chemical Treatment
}

\author{
Norsuzailina Mohamed Sutan ${ }^{1, *}$, Siti Masjida Mazlan ${ }^{1}$, Siti Noor Linda Taib ${ }^{1}$,Delsye Teo \\ Ching Lee ${ }^{1}$, Alsidqi Hassan ${ }^{1}$, Siti Kudnie Sahari ${ }^{2}$, Khairul Anwar Mohamad Said ${ }^{3}$, and \\ Habibur Rahman Sobuz ${ }^{4}$ \\ ${ }^{1}$ Civil Engineering Department, Universiti Malaysia Sarawak, 94300 Kota Samarahan, Sarawak, \\ Malaysia \\ ${ }^{2}$ Electrical and Electronic Engineering Department, Universiti Malaysia Sarawak, 94300 Kota \\ Samarahan, Sarawak, Malaysia \\ ${ }^{3}$ Chemical Engineering and Energy Sustainability Department, Universiti Malaysia \\ Sarawak, 94300 Kota Samarahan, Sarawak \\ ${ }^{4}$ Department of Building Engineering and Construction Management, Khulna University of \\ Engineering and Technology (KUET), Khulna, Bangladesh
}

\begin{abstract}
A growing interest of sugarcane bagasse fibre composite has been observed in recent years due to its attractiveness properties such as low specific weight, renewable source and producible with low investment at low cost. However, these materials have a low interfacial adhesion between fibre and matrix which lead to reduction in certain mechanical properties of the composite. To overcome this problem, studies show that certain chemical treatments on the surface of the fibres are some alternatives that significantly increase the adhesion reinforcement/matrix, in some cases improving its mechanical properties. The objective of this study was to evaluate the effect of different type of chemical treatment which are alkali and acid treatment on sugarcane bagasse fibre surface morphology. Seeking to improve the adhesion fibre matrix, the fibre has been treated with $5 \%$ of $\mathrm{NaOH}$ and $5 \%$ of $\mathrm{HCL}$ solution with added of bagasse fibre used in the range of $0 \%$ to $3 \%$ of cement weight respectively. Through SEM investigation, it is confirmed that chemical treatment helps to remove hemicelluloses from raw bagasse fiber as well as improved fibre matrix adhesion.
\end{abstract}

\section{Introduction}

Nowadays, composites have become an essential part and used by us almost every day. In the sense of being high performance materials and costs effective, lead composite to be used in broader volume in various engineering structures with ultra difficult applications such as spacecraft. Owing to the fact that in the aeronautics industry, the main goal of using composite material is the 'reduction of weight combined with strength' or 'the reduction of weight and saving precious fuel'. The uses of nature have been applied for million years by ancient society in order to change heterogeneous materials combining with the best aspects

\footnotetext{
* Corresponding author: msnorsuzailina@unimas.my
} 
of different constituents.

Despite its exorbitant price, fibre reinforced composites materials have gained popularity as it has capability to achieve high strength [1]. It has been used broadly in rehabilitation and repair of damaged masonry and concrete structures due to its high strength to weight ratio, corrosion resistance and ease of application [2]. Generally, fibres divided into two groups such as man-made fibre and natural fibre. Natural fibre is subdivided based on their origins like plants, animals or minerals. As for man-made fibres, it classified by regenerate fibres in such a way that natural materials brought into fibres form by a chemical reaction or a physical action, fibres made from synthetic polymers (synthetic fibres) and inorganic fibres [3].

In recent years, the natural fibres composites have attracted the attention of scientists due to its substantial importance as competitive and greatly potential structure of materials. Fibrous material is an advanced composite material embedded in a resin matrix, generally laminated with fibers oriented in alternating directions to give material strength and stiffness. It is originating from annual plants and has been used in broad variety of purposes. The most common natural fibres product is bagasse fibres. Bagasse is bio product of sugar cane fibres which is left after the juice has been extracted from the sugar cane. From the analysis of bagasse composition indicates that the main constituent are cellulose, hemicelluloses, lignin, ash and wax [4]. Thus, these compositions of sugarcane bagasse possess distinct physical and chemical properties make it an ideal candidate to be considered for value-added applications such as reinforcement in plastic composites for the purposes of creating new materials. Besides that, the utilization of bagasse fibres promotes low production cost with such a high quality of green products.

It cannot be denied that the merger of natural fibres into synthetic polymers helps to enhance mechanical properties. However, the major shortcomings of using natural fibres are their high degree of moisture absorption and poor dimensional. This statement is true when subjecting the use of bagasse fibres. In fact, bagasse fibres have not been subjected to any chemical surface treatments and thus contribute to weak interface between the bagasse and the resin matrix. According to a investigation by [5] in [6] research noted that the high content of lignin ( $21 \%$ on average) in sugarcane bagasse is the reason why this happened. The author also highlight that high lignin content is considered to prevent a good surface wettability between polymer matrix material and natural fibres [7] although it increases the resistance to chemical and microbial attack [8]. Therefore, a good bond between the polymeric matrix and the fibres are vital as it is responsible to transfer the load to the stiff fibres through shear stresses at the interface. Modifying the fibres surface by using chemical treatment can enhance bond strength between fibres and matrix. Chemical modifications are considered to optimize the adhesion between the fibres surface and the polymer matrix may not only modify the fibres surface but also increase fibres strength. Besides that, water absorption of composites will be reduced and enhanced their mechanical properties.

In the present study is focused on the effect of applying the different type of chemical modifications on bagasse fibres for use in natural fibres reinforced composite. Chemical treatments include alkali and acid are discussed and compare with untreated fibres. Apart from that, the benefits of using the surface treatment process and how it can improve and change the properties of fibres and composites are also discussed. Then changes in cement composite properties investigated were then related to varying the percentage of bagasse fibres proportions subjected to different chemical treatments. 


\section{Materials}

Sugarcane bagasse (Saccharum Officinarum) is the fibrous solid residue left over from the extraction of sugarcane juice. It was collected from the juice shop after they were crushed by using a hand crushing machine. It is white in colour with a length of $100-300 \mathrm{~mm}$ and free from hard, thick outer layer. After that, the collected bagasse is allowed to dry under sunlight for 2 to 3 days and spread on a waterproof sheet in order to remove the wetness that present in it. The bundled fibres were peeled out and separated manually by means of scissors. For the untreated fibres, the fibres are cut into dimension of approximately 10 $\mathrm{mm}$.

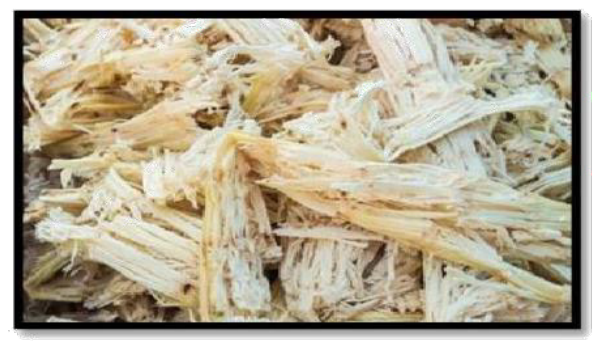

Fig. 1. Raw Bagasse Fibre.

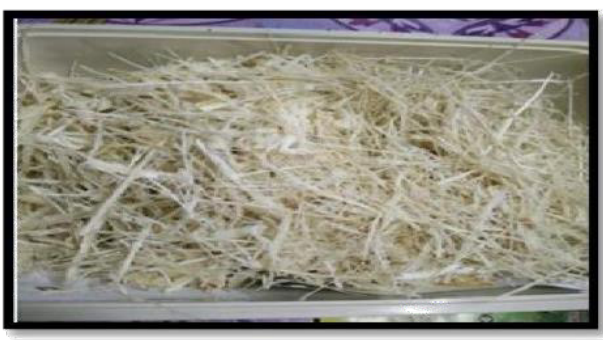

Fig. 2. Bagasse fibres that has been peeled out.

Table 1. Average Bagasse composition [6].

\begin{tabular}{|c|c|}
\hline Items & Percentage (\%) \\
\hline Moisture & 49 \\
\hline Soluble solids & 2.3 \\
\hline Fiber & 48.7 \\
\hline Cellulose & 41.8 \\
\hline Hemicellulose & 28 \\
\hline Lignin & 21.8 \\
\hline
\end{tabular}

\section{Methods}

\subsection{Fibre treatment}

Once the fibres has been prepared then it has undergone the surface treatments. The treatment of bagasse fibre was performed by alkaline treatment and acid treatment with similar concentration solution. For alkaline treatment, the dry fibres were treated with 5\% by mass of $\mathrm{NaOH}$ solution while the acid treatment was carried out in a solution of $5 \%$ by mass of HCL. The bagasse fibres were soaked for 1 hour and it has been taken out and washed several times with fresh water to make sure all the water soluble chemicals and by products were removed. Next, the bagasse fibres washed again with distilled water until final $\mathrm{pH}$ of 7 was maintained. The fibres were then dried at room temperature for 48 hours followed by oven drying at $100^{\circ} \mathrm{C}$ for 6 hours. After pretreatment reaction, the fibres were cut into the dimensions with length of approximately $10 \mathrm{~mm}$ to be mixed with cement. This was done for acid, alkaline and untreated fibres. Then it was stored in sealed plastic bag to prevent it from moisture sorption from surrounding air and moist. Fig. 3 shows the soaking fibres into alkaline and acid solution. 


\subsection{Production of cement composite}

The amount of bagasse fibres was the major parameter of this study. These fibres were in used in the range of $0 \%$ to $3 \%$ of cement weight respectively as shown in Table 2 . Composite cements were designed and made with w/c ratio of 0.42 which is the suitable content used in the cement mixture. Before the mixing procedure is conducted, bagasse fibres with various percentage of $1 \%, 2 \%, 3 \%$ (by weight relative to cement weight) of short fibres of $10 \mathrm{~mm}$ in length, with random fibres orientation, were prepared from the untreated and treated fibres. In order to produce a cement paste, particular amount of cement weight and water were mixed together. Then, particular amount of bagasse fibres is added into the cement paste and mixed well with a spatula to obtain homogenous paste. The mixture was then poured into a cylinder moulds in order to be introduced to make paste samples. Crushing of the cubes was carried out at 7,14, 28 and 56 days respectively to determine the compressive strength of the composite.

\subsection{Morphological and physical characteristics of the fibres}

\subsubsection{Scanning Electron Microscopy (SEM)}

To characterize the morphology of untreated and treated fibres surfaces and the mode of the material removal, the samples were observed under a scanning electron microscopy (SEM). The SEM used in this experiment is JEOL JSM-6390 microscope which is located at Mechanical and Manufacturing laboratory, UNIMAS. In this research, SEM will be conducted before and after fibre treated and when the fibre mixed with cement paste. Right after the sample failed undergone compressive strength; small piece of sample with the size of $1 \mathrm{~cm} \times 1 \mathrm{~cm}$ is polished, coated with thin layer of gold then stored in a sealed plastic bag before it is taken to conduct SEM. The samples are magnified up to 500 times in order to allow the submicron scale features of the samples to be seen.

\section{Results and discussions}

\subsection{Effect of fibre treatment on physical characteristics}

\subsubsection{Colour of Bagasse Fibre}

As mentioned previously, two different procedures for surface modifications were applied to bagasse fibres which are alkali treatment and acid treatment. As a result of applying chemical treatment on bagasse fibres can be seen clearly by the colour changes after treatment. The colour of treated bagasse fibres is visible in Figure 3 below. It can be seen that the appearances of the bagasse fibre changes after the chemical treatment take place. After treated with $\mathrm{NaOH}$ solution in 1 hour, it has been observed that the sugarcane bagasse fibres turns into yellowish in colour. At this point, the $\mathrm{pH}$ increase as high as $\mathrm{pH} 9 \sim 10$, which means the fibre is alkalizing and turns the colour to become even more yellowish as illustrated in Figure 3(a). This may stem from the removal of the impurities that exist on the surface of the fibre. Furthermore, the surface of the fibre becomes smoother and a bit sticky when it soaked into $\mathrm{NaOH}$ solutions compared to untreated and acid bagasse fibre.

Basically, raw bagasse fibre has impurities that accumulated on the surface of the fibre during harvest, transportation and storage after the extraction of the juice. They may contain dust, soil and other debris. These impurities however can be removed from the surface by fibre treatment. How the way it removes can be seen clearly based on the colour 
change. In this case, the finding provides evidence that there is a significant change of colour that leads to removal of impurities on the surface of the fibre become more yellowish. This is supported by [9] in his study on the effect of alkali treatment on properties of kenaf fibre. The author found that the changing of the colour of fibre treated with alkali to yellowish may be due to the removal of waxy layer and impurities from the fibre surface. This finding is similar to bagasse fibre as it turns to yellowish. Similarly, the author found that after kenaf fibre treated with alkali treatment, the surface become rather smoother as compared to that of untreated kenaf fibre.

In addition, according to [9], it was found those higher $\mathrm{pHs}$ are more effective to modify the fibre structure to obtain better mechanical properties. The efficiency in removing lignin, wax and oils that cover the external surfaces of the cell wall is higher when treated with alkali treatment. Based on the physical observation, the surface of the fibre becomes smoother, clean and a bit sticky when it soaked into $\mathrm{NaOH}$ solutions compared to untreated and acid bagasse fibre. Under the circumstances, the author reported that there is a possibility of interfacial interaction between the cement matrix and the reinforcing fibre. Next, the increase of effective surface area causes the possibility of contact between the fibre and cement matrix to occur. Plus, it also indicates that the possibility of loading transfer between the fibre and cement matrix. Hence, the surface texture would increase the number of possible interactions thereby improve mechanical fibre matrix adhesion. Meanwhile, for the case of acid treatment, no color change is identified for HCL treated fibre. This is because acid solution is not substantive to cellulosic fibres.

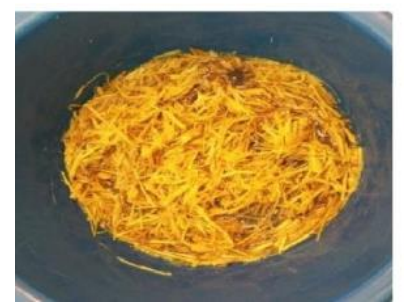

(a)

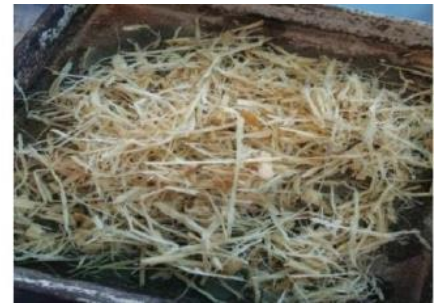

(b)

Fig 3. Bagasse fibre after treated in solution after 1 hour (a) $5 \% \mathrm{NaOH}$ treated, (b) $5 \% \mathrm{HCL}$ treated.

\subsubsection{Thickness dimension}

The effect of chemical treatments of bagasse fibre on the thickness dimension was examined using treated fibre composites. The discussion of the results begins with alkali treatment after being heated in oven for 6 hours in $100{ }^{\circ} \mathrm{C}$. As seen in Fig. 4 , the greatest decrease in diameter of the bagasse fibre was observed for $5 \% \mathrm{NaOH}$ treated fibre. This happened because of the removal of hemicelluloses and lignin that cause the surface of the bagasse fibre become smooth. Thus it leads to a decrease in diameter and fibrillation of the fibre bundles into small fibre. Even though it decreases the thickness dimension but it promotes the crystallinity of the fibre [10]. However for acid treatment not much different in terms of thickness dimension are observed. The dimension still the same like the untreated but acid fibre have shining effect and it does not fibrils into small fibre just like alkali fibre. 


\subsection{Surface Morphology of fibre}

\subsubsection{Alkali Treatment}

To confirm the effect of alkali treatment using $5 \% \mathrm{NaOH}$ solution on morphological characteristics of bagasse fibres, the samples were studied using SEM. Fig. 4 shows that differences are apparent in surface morphology between untreated and alkali treated fibres. Fig. 4 (a) show the SEM images of untreated bagasse fibre. The results indicate that the superficial layer of bagasse fibre is evident and the filaments in the untreated fibre were still packed together. In other words, the micrograph of untreated fibre looks very rigid and compact. These findings reveal that, on the surface of the fibre, the cellulose microfibrils were still intact with a layer of fatty tissue and sugarcane lignin [10].

The alkali treatment has been investigated by many researchers as one of the most effective pre treatment methods which predominantly eliminate lignin and hemicelluloses on fibres surface. A significant change in the morphological structure of the bagasse fibres occurred after treated with $5 \% \mathrm{NaOH}$ for 1 hour is illustrated in Fig. 4 (b). The fibrils were starting to separate from each other and breaking down the untreated fibres into smaller parts This happened due to the dissolution of hemicelluloses that plays a major role in removing bagasse surface from impurities, wax and hemicelluloses structure. As a result, the diameter of fibres diameter decreases with a highly fibrillated surface or an increase of the surface area.

The most striking result to emerge from this finding is that the effect alkaline solution manages to removed lignin or other cementing components. As a consequence, surface area becomes increase and helps to improve fibre-matrix adhesion in composite [10-11]. In another study [12-14] research, emphasize on the effect of $\mathrm{NaOH}$ pretreatment of lignocellulosic materials. The author observed that this treatment cause swelling and thus leading to an increase in internal surface area and a decrease in the degree of polymerization and crystallinity. As highlighted by the author, $\mathrm{NaOH}$ treatment is more effective to enhance the enzymatic digestibility compared to acid treatment due to the fact that alkali has a stronger ability in de-lignification. On the other hand, if prolong the duration of the treatment, the rough and degraded surface on fibres skeleton reflected the partial removal of crystalline cellulose material.

\subsubsection{Acid Treatment}

Researchers have studied the effects of acid treatments on vegetable fibres. It was found that it varies with the nature and concentration of the acid and temperature of the experiment [15]. In this kind of treatment, the hemicelluloses are easily hydrolyzed by acids and later form of their correspondent monomer elements. Fig. 4(c) shows the effects of an acid attack on bagasse fibres. From the SEM observation, it is apparent that the skeleton of the fibres and its surrounding layer are partly destroyed. Therefore, the fibre become weaker. 


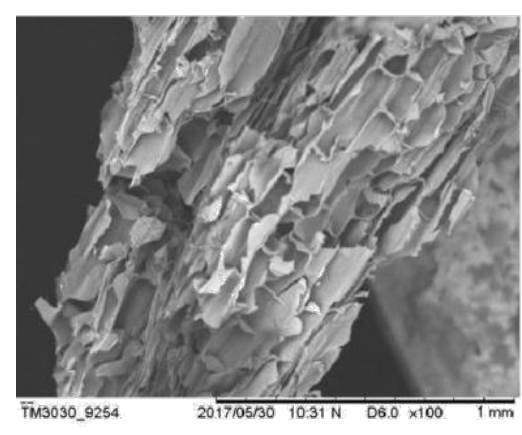

(a)

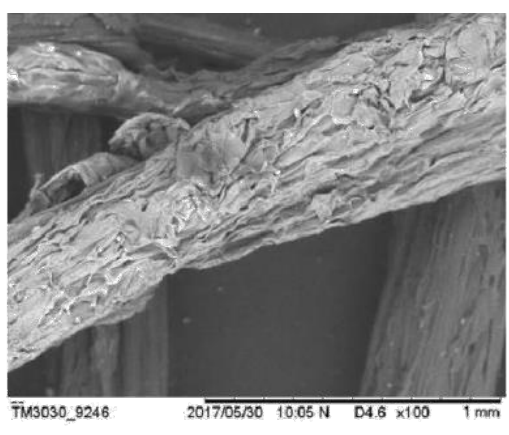

(b)

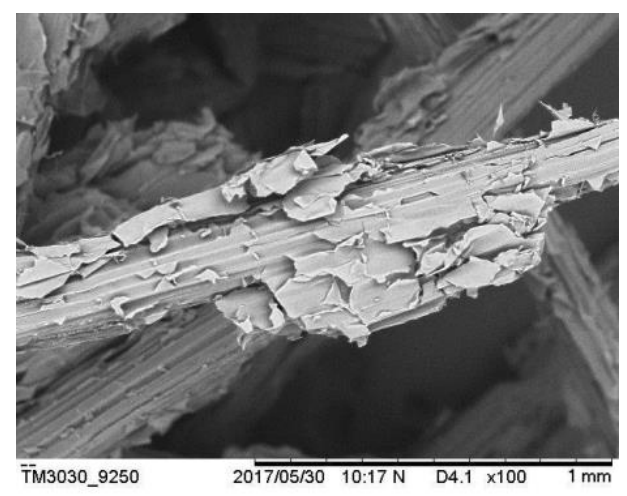

(c)

Fig. 4. SEM micrographs of bagasse fibre (a) Untreated fibre, (b) Alkali treated bagasse fibre, (c) Acid treated bagasse fibre.

\section{Conclusions}

Based on this study natural fibre can potentially provides satisfactory solution in terms of economical and environmental concerns regarding waste management. By modifying the fibre surface by using chemical treatment can enhance bond strength between fibre and matrix. Alkali treatment is more effective in removing hemicelluloses, lignin and wax from fibre surface by making it rougher as compared to acid attack. This was confirmed by SEM micrograph analysis. The treatment promotes better fibre matrix interface and improved mechanical properties of cement paste.

The authors wish to acknowledge Ministry of Higher Education and University Malaysia Sarawak for supporting this work under FRGS/TK04(02)/1138/2014(05)

\section{References}

1. Agarwal, B. D., Broutman, L. J., \& Chandrashekhara, K., Analysis and Performance of Fiber Composites (Wiley, 2006)

2. Natali, A., Manzi, S., \& Bignozzi, M. C., Procedia Engineering 21, 1124-113 (2011)

3. Bartl, A., Mihalyi, B., \& Marini, I., Chemical and Biochemical Engineering 18(1), $21-$ 28 (2004). 
4. Loh, Y. R., Sujan, D., Rahman, M. E., \& Das, C. A., Resources, Conservation \& Recycling 75, 14-22 (2013)

5. Cadenas, G. A., Mitrani, R. B., Pena, C. G., Munilla, M. H., and Correa, J. L., In: Silverio, H. N. (ed.) Manual de los Derivados de la Cana de Azucar, 2nd Ed. (in Spanish). GEPLACEA, Mexico City, Mexico, 47 (1990)

6. Acharya Samir Kumar, Mishra Punyapriya, \& Mehar, S. K., BioResources 6(3), 31553165 (2011)

7. Navarro, R. F., Medeiros, J., Mariz, T. F., and Maia, D. F. Determination of cellulose and lignin content from luffa cylindrical fibers (in Portuguese), Proceedings of the 1st Brazilian Polymer Conference, Sao Paulo, Brazil 2, 679-681 (1991)

8. Godfried, L. M. Unsaturated Polyester Resin. Its Production and Reinforcement with Natural Organic Fibers, (Fokker-VFW B.V. Report, The Netherlands, 1975)

9. Lam, T. F., \& Yatim, J. M., IJRET: International Journal of Research in Engineering and Technology, 37-40 (2015)

10. A., N. R. Juliana, Alkali treatment on sugarcane bagasse to improve properties of green composites of sugarcane bagasse. Ceramic Engineering and Science Proceedings 35(2), (2014)

11. A. E Milena, C. G. Antonio, J. E. B. Freitas, P. B. A. Fechine, A. S. B. Sombra, E. Corradini, G. Mele, A. Maffezzoli, S. E. Mazzetto, Bioresources 5(4), 2478-2501 (2010)

12. Y. Sun, J. Cheng, Bio-resource Technol 83, 1-11 (2002)

13. Y. Sudiayani, S. Alawiyah, K. Sembiring, Menara Perkebuan 78(2), 70-74 (2010)

14. N.M. Sutan, S. Hamdan, H.R. Sobuz, V.Laja, M.S. Islam, Concrete Research Letters 2(4), 326-335 (2012)

15. Arsène, M.-A., Bilba, K., Savastano Jr, H., Ghavami, K, Materials Research 16(4), 903-923. (2013) 